\title{
THE TWO-PARAMETER STURM-LIOUVILLE PROBLEM FOR ORDINARY DIFFERENTIAL EQUATIONS. II
}

\author{
B. D. SLEEMAN ${ }^{1}$
}

\begin{abstract}
The object of this note is to discuss the existence under fairly general conditions of solutions of the two-parameter eigenvalue problem defined by the pair of differential equations
\end{abstract}

$$
d^{2} y_{i} / d x_{i}^{2}+\left\{q_{i}\left(x_{i} ; \lambda, \mu\right)+r_{i}\left(x_{i}\right)\right\} y_{i}=0, \quad i=1,2, x_{i} \in[0,1],
$$

and associated two-point Sturm-Liouville end conditions.

1. Introduction. The problem discussed in this note is a continuation of the investigations already carried out by the author in a previous paper [1] bearing the same title. In [1] we considered the problem defined by

$$
\begin{aligned}
& d^{2} y \mid d x^{2}+\{q(x ; \lambda, \mu)+r(x)\} y=0, \quad x \in[a, c], a<b<c . \\
& \cos \alpha_{1} y(a)-\sin \alpha_{1} y^{\prime}(a)=0, \\
& \cos \alpha_{2} y(b)-\sin \alpha_{2} y^{\prime}(b)=0, \quad \alpha_{r} \in[0, \pi), r=1,2,3,,^{\prime} \equiv d / d x . \\
& \cos \alpha_{3} y(c)-\sin \alpha_{3} y^{\prime}(c)=0,
\end{aligned}
$$

By imposing fairly weak conditions on the coefficients $q(x ; \lambda, \mu)$ and $r(x)$ of $y$ it was shown that it is possible to choose the parameters $\lambda, \mu$ such that there exists a nontrivial solution $y(x ; \lambda, \mu)$ which not only satisfied the conditions (1.2) but also has a prescribed number of zeros in each of the intervals $(a, b),(b, c)$.

This note applies the analysis contained in [1] to the following system.

$$
\begin{aligned}
d^{2} y_{i} / d x_{i}^{2}+\left\{q_{i}\left(x_{i} ; \lambda, \mu\right)+r_{i}\left(x_{i}\right)\right\} y_{i}\left(x_{i}\right) & =0, \\
\cos \alpha_{i} y_{i}(0)-\sin \alpha_{i} d y_{i}(0) / d x_{i}=0, & \alpha_{i} \in[0, \pi), \\
\cos \beta_{i} y_{i}(1)-\sin \beta_{i} d y_{i}(1) / d x_{i}=0, & \beta_{i} \in(0, \pi], \\
& i=1,2, x_{i} \in[0,1] .
\end{aligned}
$$

Received by the editors December 23, 1970 and, in revised form, April 12, 1971.

AMS 1970 subject classifications. Primary 34B10; Secondary 34B25.

Key words and phrases. Two-parameter Sturm-Liouville problem, existence, number of zeros of solution.

1 The research for this paper was supported by the Air Force Office of Scientific Research under Grant No. AFOSR-71-2013. Reproduction in whole or part permitted for any purpose of the U.S. Government.

(c) American Mathematical Society 1972 
2. Preliminaries. We have the following lemmata [1],

LEMMA 1. Denote by $I, J$ intervals of the real line. Let

(i) $q(x ; \lambda)$ be a continuous function of $x \in I$ and $\lambda \in J$.

(ii) $y(x ; \lambda)$ be a nontrivial solution of the differential equation

$$
d^{2} y / d x^{2}+q(x ; \lambda) y=0
$$

satisfying the condition $\cos \alpha y(0, \lambda)-\sin \alpha y^{\prime}(0, \lambda)=0$, in which $\alpha$ is independent of $\lambda$.

(iii) $\phi^{(m)}(\lambda) \in I$ be the $m$ th zero of $y(x ; \lambda)$ on the right of zero, $m \geqq 1$.

Then the function $\phi^{(m)}(\lambda)$ is continuous in $\lambda \in J$ for all $\phi^{(m)}(\lambda) \in I$.

LEMMA 2. Let

(i) $m(\geqq 1)$ be a positive integer.

(ii) $q(x ; \lambda, \mu)$ and $r(x)$ be continuous for $x, \lambda, \mu \in[0,1] \times(-\infty, \infty)$ $\times(-\infty, \infty)$ and let $q(x ; \lambda, \mu)$ be a monotonically increasing function of $\lambda$, i.e. for every $\mu \in(-\infty, \infty)$ and $x \in[0,1], \lambda_{1}<\lambda_{2}$ implies $q\left(x ; \lambda_{1}, \mu\right) \leqq$ $q\left(x ; \lambda_{2}, \mu\right)$, with strict inequality holding at a set of points of $[0,1]$ whose measure is not zero.

(iii) There exists a $\lambda^{*}(\mu)$ for each fixed $\mu \in(-\infty, \infty)$ so that a nontrivial solution $y\left(x ; \lambda^{*}(\mu), \mu\right)$ of

$$
d^{2} y / d x^{2}+\left\{q\left(x ; \lambda^{*}(\mu), \mu\right)+r(x)\right\} y=0,
$$

satisfying $\cos \alpha y(0)-\sin \alpha y^{\prime}(0)=0$, exists and has more than m-zeros in $(0,1)$.

(iv) There exists a $\lambda_{*}(\mu)$ for each fixed $\mu \in(-\infty, \infty)$ so that a nontrivial solution $y\left(x ; \lambda_{*}(\mu), \mu\right)$ of

$$
d^{2} y / d x^{2}+\left\{q\left(x ; \lambda_{*}(\mu), \mu\right)+r(x)\right\} y=0,
$$

satisfying $\cos \alpha y(0)-\sin \alpha y^{\prime}(0)=0$, exists and has less than m-zeros in $(0,1)$. Then there exists a continuous function $\lambda(\mu)$ for $\mu \in(-\infty, \infty)$ such that a nontrivial solution $y(x ; \lambda(\mu), \mu)$ of

$$
d^{2} y / d x^{2}+\{q(x ; \lambda(\mu), \mu)+r(x)\} y=0,
$$

satisfying the condition $\cos \alpha y(0)-\sin \alpha y^{\prime}(0)=0$, has precisely m-zeros in $(0,1)$ and satisfies the condition $\cos \beta y(1)-\sin \beta y^{\prime}(1)=0, \beta \in[0, \pi)$, where $\beta$ is independent of $\lambda$ and $\mu$.

Lemma 2 in the form stated here is more general than the corresponding lemma in [1] where the existence of $\lambda^{*}(\mu), \lambda_{*}(\mu)$ is not assumed. Without these assumptions $q(x ; \lambda, \mu)$ must be not only weakly increasing in $\lambda$ but must also become unbounded as $\lambda \rightarrow \pm \infty$. However with the introduction of hypotheses (iii), (iv) the proof of Lemma 2 follows along similar 
lines to that of the corresponding lemma in [1] and will not be repeated here.

Note that Lemma 2 remains true and requires only minor modification if $q(z ; \lambda, \mu)$ is a monotonically decreasing function of $\lambda$. Also, if $\beta=0$ then $y(x ; \lambda, \mu)$ has a zero at $x=1$ and hence only $m-1$-zeros in the open interval $(0,1)$.

3. The existence theorem. In this section we discuss the existence of an eigenvalue pair $\lambda, \mu \in(-\infty, \infty) \times(-\infty, \infty)$ such that the problem defined by (1.3), (1.4) has nontrivial solutions $y_{1}\left(x_{1} ; \lambda, \mu\right), y_{2}\left(x_{2} ; \lambda, \mu\right)$ such that $y_{1}\left(x_{1} ; \lambda, \mu\right)$ has $m_{1}$-zeros in $(0,1)$ and $y_{2}\left(x_{2} ; \lambda, \mu\right)$ has $m_{2}$-zeros in $(0,1), m_{1}, m_{2} \geqq 1$.

Denote by $\lambda_{1}^{m_{1}}(\mu)$ the function $\lambda$ giving rise to the nontrivial solution $y_{1}\left(x_{1} ; \lambda, \mu\right)$ of $(1.3),(1.4)$ with $i=1$ which has precisely $m_{1}$-zeros in $(0,1)$. A similar meaning is assigned to the function $\lambda_{2}^{m_{2}}(\mu)$ applied to the problem (1.3), (1.4) with $i=2$. From Lemma 2 the existence of these functions is assured.

\section{THEOREM 1. Suppose}

(i) $q_{i}\left(x_{i} ; \lambda, \mu\right)$ is a continuous function of $x_{i}, \lambda, \mu \in[0,1] \times(-\infty, \infty) \times$ $(-\infty, \infty)$ monotonically increasing (or decreasing) in $\lambda$ and let $r_{i}\left(x_{i}\right)$ be continuous in $x_{i} \in[0,1], i=1,2$.

(ii) For at least one value $\mu^{-}$of $\mu$ there exists a $\lambda^{-}$such that

$$
\begin{aligned}
& q_{1}\left(x_{1} ; \lambda^{-}, \mu^{-}\right)>0 \text { for at least one } x_{1} \in[0,1), \\
& q_{2}\left(x_{2} ; \lambda^{-}, \mu^{-}\right)<0 \text { for all } x_{2} \in[0,1] .
\end{aligned}
$$

(iii) For at least one value $\mu_{-}$of $\mu$ there exists $a \lambda_{-}$such that

$$
\begin{array}{ll}
q_{1}\left(x_{1} ; \lambda_{-}, \mu_{-}\right)>0 & \text { for all } x_{1} \in[0,1], \\
q_{2}\left(x_{2} ; \lambda_{-}, \mu_{-}\right)<0 & \text { for at least one } x_{2} \in(0,1] .
\end{array}
$$

(iv) $q_{i}\left(x_{i} ; \lambda, \mu\right)$ is such that for every fixed $x_{i}, \lambda, \mu$ with $q_{i}\left(x_{i} ; \lambda, \mu\right) \neq 0$,

$$
\frac{q_{i}\left(x_{i} ; K \lambda, K \mu\right)}{\operatorname{sgn}\left\{K q_{i}\left(x_{i} ; \lambda, \mu\right)\right\}} \rightarrow \infty \quad \text { monotonically as } K \rightarrow \pm \infty \text {. }
$$

Then there exist eigenvalues $\lambda$ and $\mu$ such that the system (1.3), (1.4) has nontrivial solutions $y_{i}\left(x_{i} ; \lambda, \mu\right)(i=1,2)$ having precisely $m_{i}(i=1,2)$ zeros in $x_{i} \in(0,1), m_{i} \geqq 1, i=1,2{ }^{2}$

Proof. From conditions (ii), (iii) we may assert that there exist positive quantities $\varepsilon^{-}$and $\varepsilon_{-}$and neighbourhoods $X_{i} \subset(0,1), i=1,2$, such

${ }^{2}$ The theorem remains valid if the inequalities in (ii) and (iii) are reversed. 
that

Let

$$
\begin{array}{ll}
q_{1}\left(x_{1} ; \lambda^{-}, \mu^{-}\right)>\varepsilon^{-} & \text {for } x_{1} \in X_{1}, \\
q_{2}\left(x_{2} ; \lambda^{-}, \mu^{-}\right)<-\varepsilon^{-} & \text {for all } x_{2} \in[0,1], \\
q_{1}\left(x_{1} ; \lambda_{-}, \mu_{-}\right)>\varepsilon_{-} & \text {for all } x_{1} \in[0,1], \\
q_{2}\left(x_{2} ; \lambda_{-}, \mu_{-}\right)<-\varepsilon_{-} & \text {for } x_{2} \in X_{2} .
\end{array}
$$

$$
\begin{aligned}
& R_{i}=\max _{x_{i} \in[0,1]}\left|r_{i}\left(x_{i}\right)\right|, \quad i=1,2, \\
& M=\max \left\{\frac{2 R_{1}}{\varepsilon^{-}} ; \frac{2}{\varepsilon^{-}} \frac{\left(m_{1}+1\right)^{2} \pi^{2}}{X_{2}^{2}}\right\}, \\
& N=\max \left\{\frac{2 R_{2}}{\varepsilon_{-}} ; \frac{2}{\varepsilon_{-}} \frac{\left(m_{2}+1\right)^{2} \pi^{2}}{X_{2}^{2}}\right\} .
\end{aligned}
$$

From condition (iv) there exists a $K>0$ such that

Since

$$
\begin{array}{ll}
q_{1}\left(x_{1} ; K \lambda^{-}, K \mu^{-}\right)>M \varepsilon^{-} & \text {for } x_{1} \in X_{1}, \\
q_{2}\left(x_{2} ; K \lambda^{-}, K \mu^{-}\right)<-M \varepsilon^{-} & \text {for all } x_{2} \in[0,1] .
\end{array}
$$

$$
q_{1}\left(x_{1} ; K \lambda^{-}, K \mu^{-}\right)+r_{1}\left(x_{1}\right)>M \varepsilon^{-}-\frac{M \varepsilon^{-}}{2}=\frac{M \varepsilon^{-}}{2} \geqq \frac{\left(m_{1}+1\right)^{2} \pi^{2}}{X_{1}^{2}},
$$

it follows that every solution of the differential equation (1.3) with $i=1$ and this $q_{1}$ has more than $m_{1}$-zeros on $X_{1}$ and that every solution of (1.3) with $i=2$ which satisfies the condition at $x_{2}=1$ for this $q_{2}$ has no zeros on $x_{2} \in[0,1]$. Similarly there exists an $L>0$ such that

$$
\begin{array}{ll}
q_{2}\left(x_{2} ;-L \lambda_{-},-L \mu_{-}\right)>N \varepsilon_{-} & \text {for } x_{2} \in X_{2}, \\
q_{1}\left(x_{1} ;-L \lambda_{-},-L \mu_{-}\right)<-N \varepsilon_{-} & \text {for all } x_{1} \in[0,1] .
\end{array}
$$

Thus for these $q_{i}(i=1,2)$ every solution of (1.3) with $i=1$ satisfying the condition at $x_{1}=0$ has no zeros on $x_{1} \in[0,1]$ and every solution of (1.3) with $i=2$ has more than $m_{2}$-zeros on $X_{2}$.

From this discussion and the existence of $\lambda_{1}^{m_{1}}, \lambda_{2}^{m_{2}}$ for every $\mu \in(-\infty, \infty)$ we conclude by virtue of the monotonic character of $q_{i}(i=1,2)$ with respect to $\lambda$ that $\lambda_{1}^{m_{1}}\left(K \mu^{-}\right)<K \lambda^{-}, \lambda_{2}^{m_{2}}\left(K \mu^{-}\right)>K \lambda^{-}$, also $\lambda_{1}^{m_{1}}\left(-L \mu_{-}\right)>-L \lambda_{-}$, $\lambda_{2}^{m_{2}}\left(-L \mu_{-}\right)<-L \lambda_{-}$. Thus we have $\lambda_{1}^{m_{1}}\left(K \mu^{-}\right)<\lambda_{2}^{m_{2}}\left(K \mu^{-}\right), \lambda_{1}^{m_{1}}\left(-L \mu_{-}\right)$ $>\lambda_{2}^{m_{2}}\left(-L \mu_{-}\right)$. Finally by recalling the existence and continuity of $\lambda_{1}^{m_{1}}(\mu)$, $\lambda_{2}^{m_{2}}(\mu)$ for $\mu \in(-\infty, \infty)$ it follows that there exists a $\mu^{*}$ such that

$$
\hat{\lambda}_{1}^{m_{1}}\left(\mu^{*}\right)=\hat{\lambda}_{2}^{m_{2}}\left(\mu^{*}\right)
$$

and the theorem is proved.

From Theorem 1 we have the following corollaries. 
CoRollary 1. Suppose (i) $P_{i}\left(x_{i}\right), r_{i}\left(x_{i}\right), s_{i}\left(x_{i}\right) \in C[0,1)$ with $P_{i}\left(x_{i}\right) \geqq 0$ and not identically zero on $x_{i} \in[0,1], i=1,2$. Further, let the zeros of $P_{i}\left(x_{i}\right)$, with the possible exception of a finite number of such zeros, coincide with the points where $r_{i}\left(x_{i}\right), s_{i}\left(x_{i}\right)$ both vanish $(i=1,2)$.

(ii) There exist parameters $\lambda_{-}$and $\lambda_{-}$such that

$$
\begin{array}{ll}
\lambda-P_{1}\left(x_{1}\right)+s_{1}\left(x_{1}\right)>0 & \text { for at least one } x_{1} \in[0,1), \\
\lambda-P_{2}\left(x_{2}\right)+s_{2}\left(x_{2}\right)<0 & \text { for all } x_{2} \in[0,1], \\
\lambda \_P_{1}\left(x_{1}\right)+s_{1}\left(x_{1}\right)>0 & \text { for all } x_{1} \in[0,1], \\
\lambda \_P_{2}\left(x_{2}\right)+s_{2}\left(x_{2}\right)<0 & \text { for at least one } x_{2} \in(0,1] .
\end{array}
$$

Then there exist constants $\lambda$ and $\mu$ such that

$$
d^{2} y_{i} / d x_{i}^{2}+\left\{\lambda P_{i}\left(x_{i}\right)+\mu s_{i}\left(x_{i}\right)+r_{i}\left(x_{i}\right)\right\} y_{i}=0 \quad(i=1,2)
$$

possesses nontrivial solutions satisfying the conditions (1.4) and such that $y_{1}$ has $m_{1}$-zeros on $x_{1} \in(0,1)$ and $y_{2}$ has $m_{2}$-zeros on $x_{2} \in(0,1){ }^{3}$

CoRollary 2. Let (i) $s_{i}\left(x_{i}\right), r_{i}\left(x_{i}\right) \in C[0,1], m_{1}, m_{2} \geqq 1$ be positive integers,

(ii) $s_{1}\left(x_{1}\right)$ attain its maximum only on $[0,1), s_{2}\left(x_{2}\right)$ attain its minimum only on $(0,1]$ (or conversely).

Then there exist parameters $\lambda$ and $\mu$ such that the differential equations

$$
d^{2} y_{i} / d x_{i}^{2}+\left\{\lambda+\mu s_{i}\left(x_{i}\right)+r_{i}\left(x_{i}\right)\right\} y_{i}=0, \quad i=1,2,
$$

possess nontrivial solutions satisfying the conditions (1.4) and such that $y_{1}$ has precisely $m_{1}$-zeros on $x_{1} \in(0,1)$ and $y_{2}$ has $m_{2}$-zeros on $x_{2} \in(0,1)$.

Equations of the type considered in the above corollaries are a subclass of the multi-parameter systems discussed by Faierman [2].

4. Examples. Because of the weakness of the conditions imposed on $q_{i}\left(x_{i} ; \lambda, \mu\right)$ in Theorem 1 , it is clear that the results of this note are applicable to a wide class of two-parameter systems. Some examples are given below.

(I) $q_{i}\left(x_{i} ; \lambda, \mu\right)=\lambda^{2} f_{i}\left(x_{i}\right)+2 \lambda \mu g_{i}\left(x_{i}\right)+\mu^{2} h_{i}\left(x_{i}\right), i=1,2$, where $f_{i}, g_{i}, h_{i}$ $\in C[0,1]$,

$$
\begin{array}{lll}
f_{1}\left(x_{1}\right)>0, & \left(g_{1}^{2}-h_{1} f_{1}\right) \geqq 0, & \forall x_{1} \in[0,1], \\
h_{2}\left(x_{2}\right)<0, & \left(g_{2}^{2}-h_{2} f_{2}\right) \leqq 0, & \forall x_{2} \in[0,1] .
\end{array}
$$

${ }^{3}$ This corollary remains true if all the inequalities in condition (ii) are reversed. 
(II) $q_{i}\left(x_{i} ; \lambda, \mu\right)=(-1)^{i+1} \cosh \lambda x_{i}+\sinh \mu x_{i}, i=1,2, x_{i} \in[0,1]$.

(III) $q_{i}\left(x_{i} ; \lambda, \mu\right)=(-1)^{i+1} \lambda+(-1)^{i} \cosh \mu x_{i}, i=1,2, x_{i} \in[0,1]$.

(IV) Consider the eigenvalue problem defined by (1.1), (1.2) in which $a, b$ are real, $c$ is complex of the form $c=b+i d$, and $\alpha_{r}=0$ or $\pi / 2, r=2,3$.

Further assume that for $\lambda, \mu$ real $q(z ; \lambda, \mu)$ and $r(z)$ are real valued for $z \in[a, b]$ and $z \in[b, c]$ where $[b, c]$ is understood to mean the straight line interval joining $b$ and $c$. The problem is then easily converted into one of the type defined by (1.3), (1.4). Systems of this type arise, for example, in the study of Lamé's equation or Mathieu's equation.

\section{REFERENCES}

1. B. D. Sleeman, The two-parameter Sturm-Liouville problem for ordinary differential equations, Proc. Roy. Soc. Edinburgh Sect. A69 (1971), 139-148.

2. M. Faierman, The completeness and expansion theorems associated with the multiparameter eigenvalue problem in ordinary differential equations, J. Differential Equations 5 (1969), 197-213. MR 38 \#1314.

Courant Institute of Mathematical Sciences, New York University, New YORK, NEW YORK 10012

Current address: Department of Mathematics, University of Dundee, Dundee, Scotland 